\title{
UTILIZATION OF MICRO SISAL FIBERS AS REINFORCEMENT AGENT AND POLYPROPYLENE OR POLYLACTIC ACID AS POLYMER MATRICES IN BIOCOMPOSITES MANUFACTURE
}

\author{
Subyakto, Nanang Masruchin, Kurnia Wiji Prasetiyo and Ismadi ${ }^{1}$ \\ Received : 20 September 2012, Accepted : 23 June 2013
}

\begin{abstract}
Sisal (Agave sisalana) as a perennial tropical plant grows abundantly in Indonesia. Its fibers can be used as the reinforcement agent of biocomposite products. Utilization of sisal as natural fiber has some notable benefits compared to synthetic fibers, such as renewable, light in weight, and low in cost. Manufacture of biocomposite requires the use of matrix such as thermoplastic polymer, e.g. polypropylene (PP) and polylactic acid (PLA) to bond together with the reinforcement agent (e.g. sisal fibers). In relevant, experiment was conducted on biocomposites manufacture that comprised sisal fibers and PP as well as PLA. Sisal fibers were converted into pulp, then refined to micro-size fibrillated fibers such that their diameter reduced to about $10 \mu \mathrm{m}$, and dried in an oven. The dry microfibrillated sisal pulp fibers cellulose (MSFC) were thoroughly mixed with either PP or PLA with varying ratios of MSFC/PP as well as MSFC/PLA, and then shaped into the mat (i.e. MSFC-PP and MSFCPLA biocomposites). Two kinds of shaping was employed, i.e. hot-press molding and injection molding. In the hot-press molding, the ratio of MSFC/PP as well as MSFC/PLA ranged about 30/70-50/50. Meanwhile in the injection (employed only on assembling the MSFC-PLA biocomposite), the ratio of MSFC/PLA varied about 10/90-30/70. The resulting shaped MSFC-PP and MSFC-PLA biocomposites were then tested of its physical and mechanical properties. With the hot-press molding device, the physical and mechanical (strength) properties of MSFC-PLA biocomposite were higher than those of MSFC-PP biocomposite. The optimum ratio of MSFC/PP as well as MSFC/PLA reached concurrently at 40/60. The strengths of MSFC-PP as well as MSFCPLA biocomposites were greater than those of individual polymer (PP and PLA). With the injection molding device, only the MSFC-PLA biocomposite was formed and its strengths reached maximum at 30/70 ratio. The particular strengths (MOR and MOE) of MSFC-PLA biocomposite shaped with injection molding were lower than those with hot-press molding, both at 30/70 ratio. The overall MOR of such MSFC-PLA biocomposite was lower than that of pure PLA, while its MOE was still mostly higher.
\end{abstract}

Keywords: Biocomposites, sisal, micro-size fibers, polypropylene, polylactic acid, physical mechanical properties

\section{INTRODUCTION}

Biocomposites or green composites have been subjected to extensive research in the last decades (Mohanty et al., 2002; Netraveli and Chabba, 2003; Berglund, 2004; Mathew et al., 2005; BogoevaGaceva et al., 2007; John and Thomas, 2008). The composites are utilized as materials for automotive components, buildings and electronics (Marsh, 2003; Nakagaito and Yano, 2005; Iwamoto et al., 2005; Bledzki et al., 2006). Composites originated from natural fibers are

${ }^{1}$ Research and Development Unit for Biomaterials - Indonesian Institute of Sciences

"Corresponding Author:subyakto@biomaterial.lipi.go.id environmentally friendly and renewable. Natural fibers are non toxic, light in weight, affording better specific mechanical properties, non abrasive to tools and cheaper in price (Mohanty et al., 2002; Oksman et al., 2003; Wambua et al., 2003; Zimmermann et al., 2004). One of the natural fibers is sisal. Research on composites made from sisal fibers has been done extensively (Joseph et al., 1999; Li et al., 2000; Arzondo et al., 2004; Mohanty et al., 2004a; Mohanty et al., 2004b). The fiber in general should be bonded together using thermosetting or thermoplastic adhesives as 
the matrix. Thermoplastic adhesives used are polypropylene (PP) and polylactic acid (PLA). The fibers, generally 1-4 $\mathrm{mm}$ in length and 20-40 $\mu \mathrm{m}$ in diameter are used for the composite manufacturing. Researches on micro-sized sisal fibers with diameter of micro size however have not been done yet. Zimmermann et al. (2004) stated that there is a high correlation between fibers size and the strength. The smaller the diameter of fibers, then the higher their mechanical properties. As an example, wood pulp fibers have modulus of elasticity (MOE) of 10 $\mathrm{GPa}$, but when in the form of crystal cellulose the MOE become $250 \mathrm{GPa}$. The smaller fibers ensure more homogenous structure, and micro crack in individual fiber which usually occurs can be avoided or minimized.

In relevant, the purpose of this research is to develop biocomposites assembled from the micro-size sisal fibers (as the reinforcement agent), mixed and bonded together with matrices of the conventional synthetic polymer (PP) as well as of natural polymer (PLA). The ratio between sisal fibers and PP as well as between sisal fibers and PLA were varied. The resulting mixtures were then shaped to a biocomposite mat using hotpress molding and injection molding devices separately. Assessment on the resulting composites was emphasized on the effect of such ratio on the physical and mechanical properties.

\section{MATERIAL AND METHOD}

\section{A. Materials}

The main materials used consist of PP, PLA, maleic anhydride modified PP (MAPP) and sisal fibers. PP was obtained from Grand Siam Composites Co., Thailand (melt flow index 13.3 $\mathrm{g} / 10 \mathrm{~min}$., melting point $165.5^{\circ} \mathrm{C}$ ); polylactic acid (PLA) Lacea H400 from Mitsui Co., Japan, triacetin and MAPP Umex 1001 from Sanyo Chemical Industries, Japan. Fibers of sisal (Agave sisalana) were obtained from Blitar area, East Java. The fibers were processed into pulp using the following treatment: at first, sisal fibers were cleaned of impurities and cut into $2 \mathrm{~cm}$ length. As much as $250 \mathrm{~g}$ (oven dried weight equivalent) was then cooked in $\mathrm{NaOH} 2.5 \%$ (1 part of fiber soaked in 10 part of solution) for 2 hours.
Afterwards, the softened fibers were separated from the cooking liquor and thoroughly washed until free of residual alkali. The fibers were then beaten into pulp using Hollander beater at about $5 \%$ consistency for 90 minutes. The resulting pulp was then refined 5 times to ensure its fiber diameter reduction. Observation by scanning electron microscope (SEM) was done before and after refining to ensure that the fiber size of pulp had reached $10 \mu \mathrm{m}$ in diameter (micro-size fiber). Afterwards the refined microfibrillated sisal pulp was stored in the freezer.

\section{B. Manufacture of Biocomposites Using Hot-Pressing Molding Devices}

\section{PP Biocomposites}

The composite as made-up of microfibrillated sisal fibers and $\mathrm{PP}$ was prepared by mixing the dry sisal pulp with PP polymer. The wet microfibrillated sisal pulp as used was the one already kept previously in the freezer. Prior to such mixing, the pulp was at first rewetting the refined pulp in water, followed by filtration. Afterward, the filtrated pulp was dried in an oven at $60{ }^{\circ} \mathrm{C}$ for 24 hours. The dried pulp was then torn-off into small-size stuffs that measured about $1 \mathrm{~cm}$ by $1 \mathrm{~cm}$, and further mixed with PP in a Laboplastomill device at $175^{\circ} \mathrm{C}$, that rotated at $60 \mathrm{rpm}$ for 20 minutes. In such mixing, the ratio between pulp fiber and PP varied, i.e. 30/70, 40/60, and 50/50 (w/w). Meanwhile, maleic anhydride modified PP (MAPP) as a coupling agent was also incorporated at constant amount ( $5 \%$ by the mixture weight). The resulting mixture (microfibrillated pulp fiber - PP composite) was shaped into the mat using a particular molding device, which measured $150 \mathrm{~mm}$ (length) by 50 $\mathrm{mm}$ (width) by $3 \mathrm{~mm}$ (thickness), and then undergone the hot-pressing molding at $180^{\circ} \mathrm{C}$ and $1 \mathrm{MPa}$ pressure for 30 seconds. Afterwards, the microfibrillated fiber-PP mat was immediately removed from the hot-pressing device, and then cold-pressed at $1 \mathrm{MPa}$ for 5 minutes. Thereafter, the cold pressing device was disengaged, and the microfibrillated fiber-PP composite was removed and conditioned at room temperature for 3 days. After being conditioned, the microfibrillated fiber-PP composite mat was tested for physical and mechanical properties in accordance with the ASTM Standards (D790), i.e. density, MOR and 
MOE (in bending).

\section{PLA Biocomposites}

In another work, the microfibrillated fiberPLA composite was prepared by mixing the wet sisal pulp fiber with such polymer (PLA) and triacetin. First, PLA was initially soaked in dichloromethane. Afterwards, the soaked PLA was added with the wet pulp (taken from pulp stored in the freezer) and triacetin, and then mixed until becoming homogenous. In such mixing, the ratio between microfibrillated pulp fiber and PLA varied similarly to that of pulp fiber and PP (i.e. 30/70, 40/60, and 50/50 in w/w). Meanwhile, the triacetin functioned as plasticizer, and the amount as mixed was constant (7\% by the mixture weight). The resulting mixture (microfibrillated pulp fiber - PLA composite) was then dried in an oven at $60^{\circ} \mathrm{C}$ for 6 hours. The dry composite was further processed in the Laboplastomill device as well at $170^{\circ} \mathrm{C}$, rotating at $60 \mathrm{rpm}$ for 20 minutes until becoming homogenous. The homogenous microfibrillated pulp fiber-PLA composite was then shaped into the mat, which measured $150 \mathrm{~mm}$ by $50 \mathrm{~mm}$ by 3 $\mathrm{mm}$ as well also using a molding device, and then undergone further stages similar those of microfibrillated pulp fiber-PP composite mat (e.g. hot pressing, cold pressing, conditioning, together with each of the variables as employed such as amount of pressure, temperature, duration, humidity). Similar to the previous composite, the microfibrillated sisal pulp fiber-PLA composite mat was tested as well of its physical and mechanical properties followed the ASTM standards (D790); and the tested properties similar to those of microfibrillated sisal pulp fiber - PP composite mat.

\section{Manufacture of PLA Biocomposites Using Injection Molding Device}

In addition to assembling the microfibrillated sisal fibers-PLA biocomposites using the hotpressing molding device, such biocomposite was also prepared by the so-called injection molding. As such, the preparation of the composite components (i.e. microfibrillated sisal pulp fibers, PLA, dchloromethane, and triacetin) and mixing procedure (i.e. sisal fiber and PLA) were similar to that employing the hot-press molding device. In such mixing, the ratio between microfibrillated sisal fibers - PLA (w/w) was varied at 10/90, $20 / 80$, and $30 / 70$. Further, different from the hot-pressing treatment, in this regard the microfibrillated sisal fibers-PLA mixture was each processed (crushed) into the pellets using a pelletizer device. Afterwards, the resulting pellets were injected into the injection molding machine (Nissei Plastic Industrial Co. Ltd. Japan, PS60E9ASE), and there inside shaped into a composite mat. The size of the mats was similar to that employing the hot-pressing, i.e. $150 \mathrm{~mm}$ by $50 \mathrm{~mm}$ by $3 \mathrm{~mm}$. The conditioned as employed during the injection molding treatment was injection temperature $140-150{ }^{\circ} \mathrm{C}$, injection pressure $45-50 \%$ (of its maximum pressure capacity), injection speed $30-40 \%$ (of its maximum speed), injection duration 10 seconds. The resulting composite samples were examined of its physical and mechanical properties in accordance with the ASTM standards (D790, D638, D785), i.e. flexural strength and flexural modulus (MOR and MOE in bending, respectively), tensile strength, tensile modulus, and hardness. Three boards were made for each treatment.

\section{RESULT AND DISCUSSION}

\section{A. SEM Observation on Microfibrillated Sisal Fibers}

SEM observations on sisal pulp fibers before and after refining are presented in Figure 1. It can be seen that after refining the fibers is more fibrillated than before. In average, the diameter of sisal fiber was around $10 \mu \mathrm{m}$, and therefore regarded as the micro-scale fibers. Almost similar work was performed by Nakagaito and Yano (2004) to obtain the nano-size diameter of cellulose fibers.

\section{B. Physical and Mechanical Properties of Biocomposites Shaped Using Hot-Pressing Molding Device}

The resulting data about density, MOR, and 

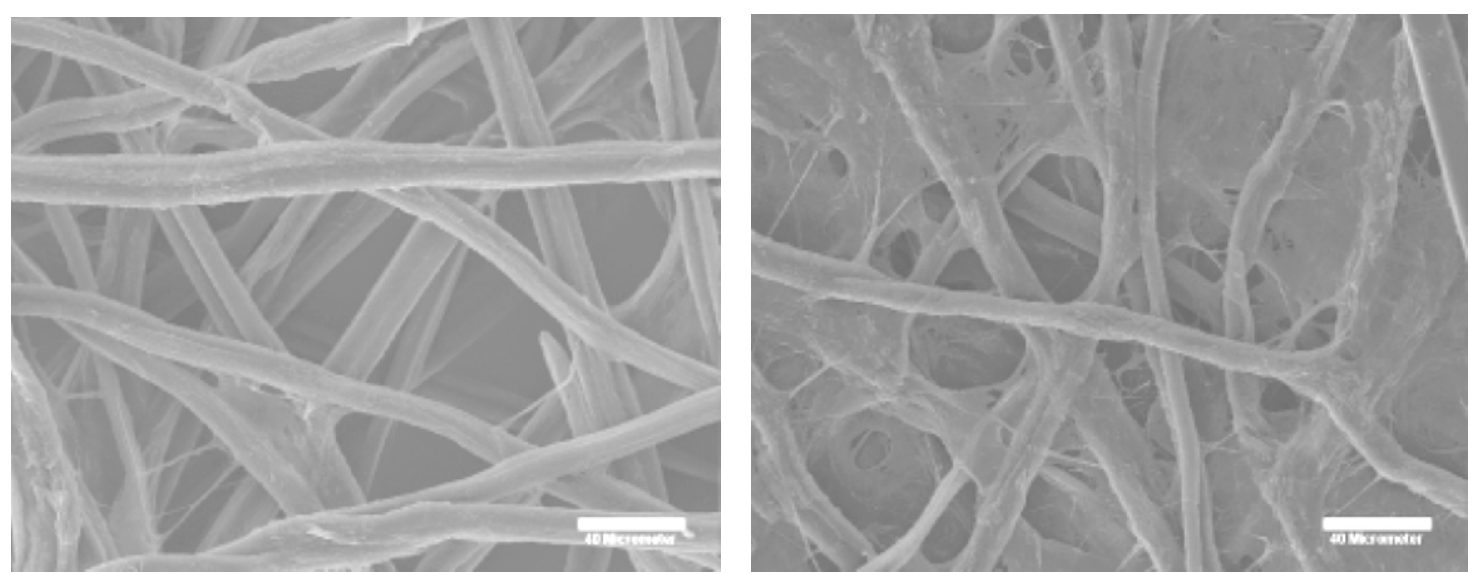

Figure 1. The SEM images on sisal pulp fibers, before refining (left), and after refining action (right)

MOE of biocomposites comprising microfibrillated sisal fiber cellulose (MSFC) and PP polymer as well as MSFC and PLA polymer are presented at Figures 2, 3, and 4, respectively. The density of biocomposites made up of PP as well as PLA matrix reinforced by the MSFC was higher than that of pure polymers (PP and PLA). This could be attributable to the higher density of sisal pulp fibers, i.e. $1.33-1.50 \mathrm{~g} / \mathrm{cm}^{3}$ (Munawar, 2008), than that of PP and PLA (i.e. $0.90 \mathrm{~g} / \mathrm{cm}^{3}$ and 1.25 $\mathrm{g} / \mathrm{cm}^{3}$, respectively). The density of MSFC-PLA biocomposite was entirely higher than that of MSFC-PP biocomposite. This could due to the presumption that PLA polymer exhibited greater polarity than PP polymer. This situation can impart more intensive hydrogen $(\mathrm{OH})$ bonding, in addition to the weak adhesion bond (van der Waals' force), in the MSFC-PLA bond than that in the MSFC-PP bond, thereby exerting more dense and compact structure inside the MSFC-PLA biocomposite. Further, the density of MSFCPLA biocomposite and MSFC-PLA biocomposite seemed insignificantly affected, regardless of the changing ratio of $\mathrm{MSFC} / \mathrm{PP}$ as well as of MSFC/PLA, i.e. 30/70, 40/60, and $50 / 50$. Greater polarity of PLA seemed also responsible for the greater density of pure PLA than that of pure PP (Figure 2). This implied that more compact/dense structure occurred to the inside PLA than to the inside PP. This is because such polarity enhanced lateral hydrogen bonding between PP polymer chains, rather than the weaker van der Waals' bond that only existed between PP polymer chains, despite being fortified by the MAPP's coupling agent.

The MOR and MOE of MSFC-PP biocomposite as well as MSFC-PLA biocomposite were higher than those of pure PP and PLA polymers (Figures 3 and 4). This seemed consistent with the greater density of those two

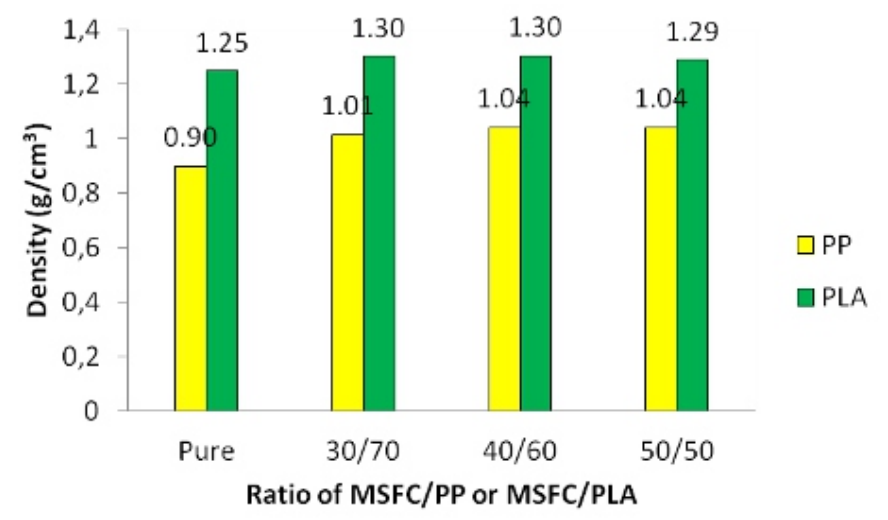

Figure 2. Density of MSFC-PP biocomposite and MSFC-PLA biocomposite

Remarks: $\mathrm{MSFC}=$ microfibrillated sisal pulp fibers; $\mathrm{PP}=$ polypropylene polymer as matrix in the MSFC-PP biocomposite; PLA = polylactic acid polymer as matrix in the MSFC-PLA biocomposite 


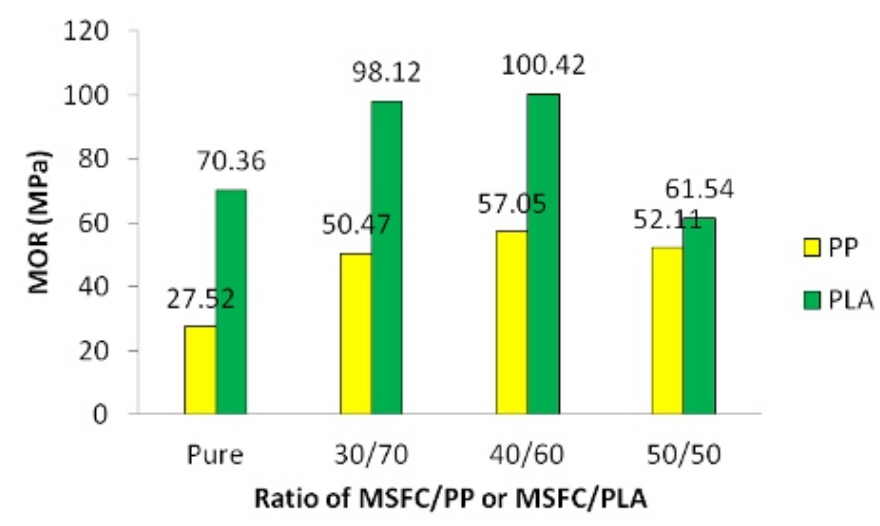

Figure 3. MOR (modulus of rupture) of MSFC-PP biocomposite and MSFC-PLA biocomposite Remarks: (see Figure 2)

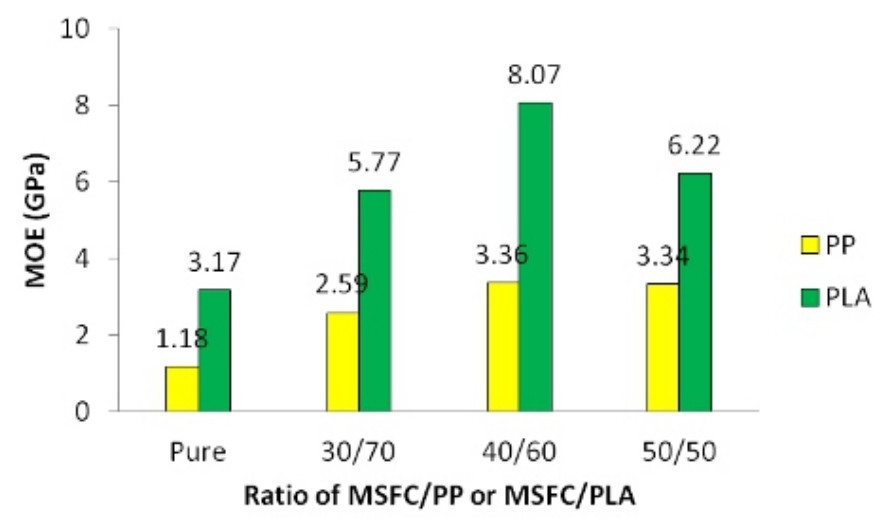

Figure 4. MOE (modulus of elasticity) of MSFC-PP biocomposite and MSFC-PLA biocomposite Remarks: (see Figure 2)

biocomposites than that of the corresponding pure PP and PLA polymer (Figure 2). Further, the MOR and MOE of pure PLA were greater than those of pure PP. This situation seemed consistent as well with the greater density of PLA than that of pure PP (Figure 2). About the MOR and MOE of the biocomposites (MSFC-PP and MSFC-PLA), they appeared to reach maximum with the mixing ratio of MSFC/PP as well as of MSFC/PLA at 40/60 (Figures 3 and 4). The explanation was that with increasing proportion of PP as well as PLA polymer from 50/50 ratio to $40 / 60$, apparently this allowed more of those two polymers (while still in melting state) each to enter into the more porous of sisal fibers and also to take-up the space between microfibrils on the fibers, thereby increasing the biocomposite strength (MOR and MOE). However, reaching the 30/70 ratio, there would be more free-mass of $\mathrm{PP}$ as well as PLA polymer in the biocomposite than that at 40/60 ratio, hence lowering the strengths instead. Further, as another explanation, the strengths (MOR and MOE) of pure PP as well as pure PLA were lower than those corresponding biocomposites (Figure 3 and 4), and therefore more portion of PP or PLA added to the could logically weaken such biocomposite strength. Conversely, the increasing portion of sisal fiber (MSFC) as the reinforcement agent in the corresponding PP as well as PLA biocomposites up to the $40 / 60$ ratio bring about positive effects on the strength. This could be tracked down to the MOE (Young's modulus) of sisal fibers, i.e. 9.038.0 GPa (Munawar, 2008) which was greater than that of pure PP or PLA (Figure 3). Apparently, with greater portion of MSFC beyond the 40/60 ratio (i.e. 50/50), less mass of PP or PLA polymer entered into the sisal fibers and/or occupied the spaces between microfibrils. This situation, despite superior strengths of individual sisal 
fibers, could create more air spaces and therefore weaken the MSFC-to-polymer-to-MSFC bonds, thereby lowering the biocomposite strength (MOR and MOE). Further, the overall strengths (MOR and MOE) of MSFC-PLA biocomposite were greater than those of MSFC-PP biocomposite (Figures 3 and 4). This could be strongly due to greater density of the former biocomposite than the latter biocomposite (Figure 2). Again, the greater polarity of PLA was responsible for the stronger MSFC-PLA bonds (contributed largely by the hydrogen-bond) than the less polarity of PP in context of weaker MSFC-PP bond (despite with the use of MAPP as coupling agent).

SEM images revealed that some fractures occurred on the surface of biocomposites, i.e. MSFC-PP composite as well as MSFC-PLA composite (Figure 5). Such fractures indicated that homogenous mixture already occurred during the mixing of MSFC and PP as well as MSFC and PLA, prior to the forming of biocomposite mat in the hot-press molding device.

Biocomposites comprising the mixture of MSFC (as reinforcing agent) and PP polymer (as matrix), added with MAPP (as coupling agent) exhibited the modulus of rupture (MOR) and modulus of elasticity (MOE) in the range of consecutively $50.47-57.05 \mathrm{MPa}$ and $2.59-3.36$ $\mathrm{GPa}$ (Figures 3 and 4). These resulting values were higher than those obtained by Mohanty et al. (2004a), who manufactured biocomposite from the mixture of sisal fiber and PP as well, but at lower ratio of sisal fiber/PP (i.e. $21 / 79$ ), i.e. $\mathrm{MOR}$ of $48.96 \mathrm{MPa}$ and MOE of $2.188 \mathrm{GPa}$. Those lower values could be attributed by two possibilities (in the experiment by Mohanty et al. 2004a), i.e. lower portion of sisal fibers in the mixture of sisal fiber and PP; the sisal fiber was not microfibrillated, instead just used the fibers as it is; and they used MAPP as coupling agent at a lower percentage (i.e. 1\%). Therefore, the use of MAPP ( $5 \%$ of mixture weight) in the assembling of MSFC-PP biocomposites in this research indicatively contributed to such optimum mixture of MSFC/PP at 40/60 (i.e. maximum strengths of MOR and MOE).

With regard to the MOR and MOE of the MSFC-PLA composite, they ranged about consecutively $61.54-100.42 \mathrm{MPa}$ and $5.77-8.07$ GPa. Research on the use of sisal fibers (as reinforcing agent) and PLA polymer (as matrix) for biocomposite apparently was not yet done before. However, for the comparison, Oksman et al. (2003) conducted research on biocomposite using flax (Linum usitatissimum) fibers and PLA. Using the ratio of flax fibers/PLA at 30/70, they obtained that tensile strength (TS) and tensile modulus (TM) reached consecutively $53 \mathrm{MPa}$ and $8.3 \mathrm{GPa}$. Further, the portion of flax fibers increased to 40/60 ratio, the mechanical strength decreased strikingly (TS at $44 \mathrm{MPa}$ and TM at 7.3 $\mathrm{GPa}$ ). These phenomena were almost consistent with those occurring in this research, where the MOR and MOE decreased with increasing portion of MSFC at the MSFC/PLA ratio exceeding 40/60 (Figures 3 and 4).
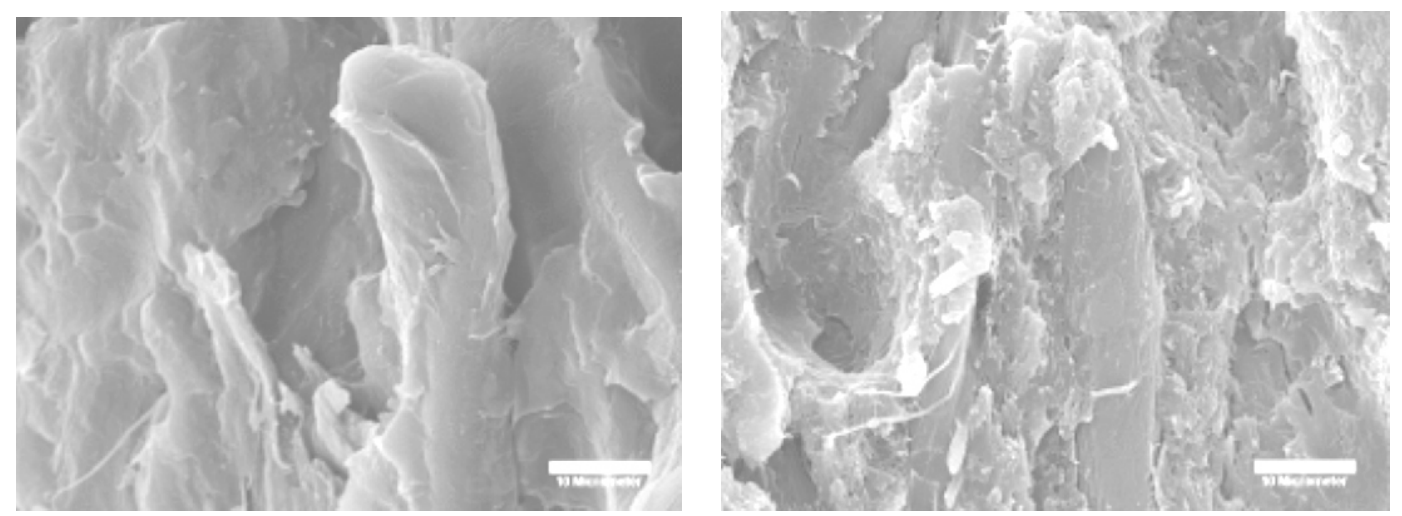

Figure 5. The SEM images revealing the fractures that occurred on the surface of both MSFC-PP biocomposite (left) and MSFC-PLA biocomposite (right), each at ratio of 50/50, shaped with the hot-press molding device 


\section{Physical and Mechanical Properties of Biocomposites Shaped Using Injection Molding Device}

Results regarding examining the physical and mechanical properties of sisal MSFC-PLA biocomposites shaped by injection manner, i.e. flexural strength (MOR), flexural modulus (MOE), tensile strength, tensile modulus, and hardness are presented in Figures 6, 7, and 8. The flexural strength (MOR) and flexural modulus (MOE) exhibited the highest values using the ratio of MSFC/PLA at 30/70, i.e. consecutively 67.83 $\mathrm{MPa}$ and $4.43 \mathrm{GPa}$. Similarly, the tensile strength of such biocomposites was also the highest at 30/70 ratio, i.e. $48.18 \mathrm{MPa}$. Meanwhile, the tensile modulus at both $30 / 70$ and $20 / 80$ ratios (MSFC/PLA) seemed similar, i.e. $1.14 \mathrm{GPa}$ vs. $1.13 \mathrm{GPa}$, respectively. It seemed that the greater portion of MSFC in the MSFC/PLA ratio tended to increase the strengths of the corresponding biocomposite (Figures 6 and 7). These phenomenon were almost consistent, similarly explainable, with those occurring with the hotpress molding device but up to the 40/60 ratio (Figures 3 and 4). Again, this situation could be attributable to greater strengths of individual sisal fibers (Munawar, 2008) compared to those of pure PLA.

The MOR and MOE of MSFC-PLA biocomposite shaped with the injection molding device, particularly at MSFC/PLA ratio of 30/70 were consecutively $67.83 \mathrm{MPa}$ and $4.43 \mathrm{GPa}$ (Figures 6 and 7). Meanwhile the MOR and MOE of the corresponding biocomposite but shaped with the hot-press molding device also at 30/70 ratio were $98.12 \mathrm{MPa}$ and $5.77 \mathrm{GPa}$, respectively
(Figures 3 and 4). It turned out that indicatively the strengths of MSFC-PLA shaped with the hotpress molding were greater than those shaped with the injection molding. This implied that the adhesion bonding (i.e. the MSFC-PLA bond occurred due to hydrogen bond and van der Waals' force) and the cohesion bond (i.e. the PLAPLA bond due to hydrogen bond and van der Waals' force as well) were more readily developed in the biocomposite shaped with the hot-press molding than those with the injection molding. This happened possibly in the injection molding, the heating energy as provided in the biocomposite-mat forming actually used for biocomposite-mat forming was partly absorbed to bring together the pellets to intimate contact and unite them into such mat. Another possibility was that the shaping of biocomposite mat from the pellets particles still allowed for the air spaces to develop inside, and therefore such could adversely affected its strengths. Further, considering that the maximum strengths of the MSFC-PLA biocomposite at 30/70 ratio, the SEM observation was conducted on the biocomposite with such ratio (Figure 9). It seemed that a lot of fractures occurred on the surface of the biocomposite, indicating that the MSFC blended homogenously with PLA.

Further, the overall MOR of MSFC-PLA biocomposite shaped using the injection molding device were lower than that of pure PLA, while its MOE was still mostly higher, except at 10/90 ratio (Figures 3, 4 and 6). This again strengthened the previous presumption that mat shaping of this biocomposite from pellet particles adversely affected the compactness of MSFC-PLA
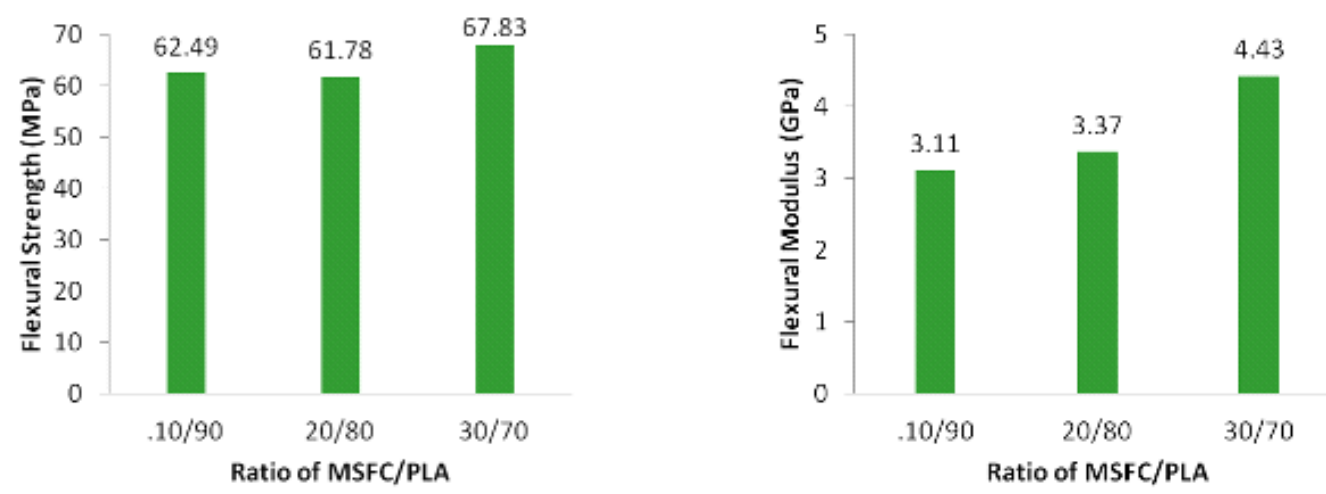

Figure 6. Flexural strength/MOR (left) and flexural modulus/MOE (right) of MSFC PLA biocomposite shaped using the injection molding device 

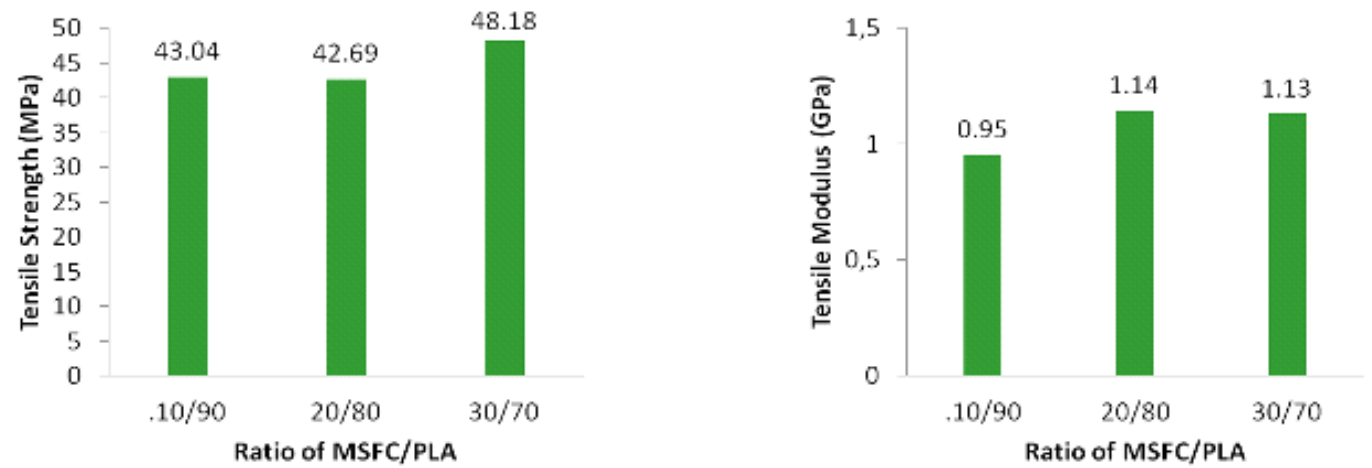

Figure 7. Tensile strength (left) and tensile modulus (right) of MSFC - PLA biocomposite shaped using the injection molding device

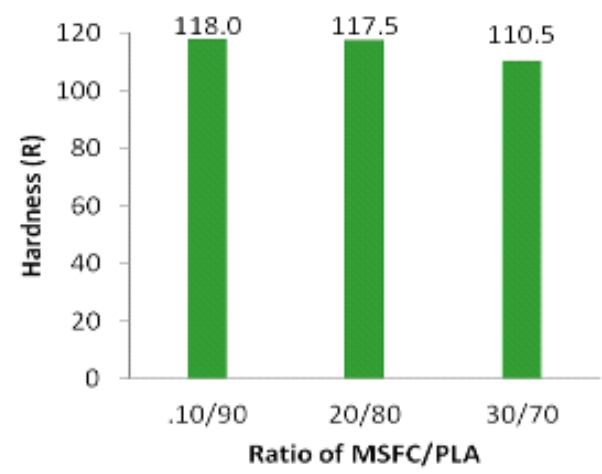

Figure 8. Hardness of MSFC-PLA biocomposite shaped using the injection molding device

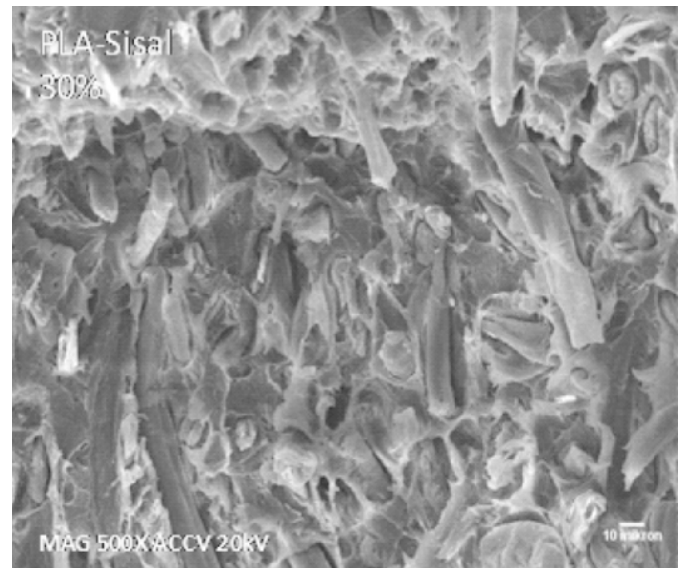

Figure 9. The SEM images revealing the fractures that occurred on the surface of MSFC-PLA biocomposites at 30/70 ratio, shaped with the injection molding device

adhesion and PLA-PLA cohesion bonds inside its structure, thereby lowering particularly its MOR.

With regard to the hardness, the MSFC-PLA biocomposite shaped with such device seemingly exhibited the greatest value at 10/90 ratio of MSFC/PLA. This implied that the most compact and dense structure of MSFC-PLA occurred inside that biocomposite at such 10/90 ratio. Greater portion of MSFC in the ratio, however, tended to decrease slightly the hardness. The possible explanation was that at such greater portion (exceeding 10/90 ratio), the amount of 
the PLA could be less which entered into the structure of microfibrillated sisal fibers and filled up the air spaces between the fibrils on the surface of those fibers.

\section{CONCLUSION}

Physical and mechanical properties of biocomposites made up of microfibrillated sisal pulp fibers as reinforcing agent and polypropylene and polylactic acid as matrices, shaped by the hotpress molding device and at ratio of fibers/ polymer ranging $30 / 70$ to $50 / 50$, was affected by kind of such polymer. The biocomposites that used PLA exhibited greater density, MOR, and MOE than that using PP. The maximum values of those three properties occurred at the biocomposite that consisted of fibers and PLA as well as fibers and and PP, concurrently at fiber/polymer ratio of $40 / 60$, or such could be regarded as the optimum ratio.

The overall physical and mechanical properties of microfibrillated sisal fibers-PLA biocomposite were greater than those of microfibrillated sisal fibers-PP biocomposite. The physical and mechanical properties of sisal fibers-PLA biocomposite were entirely greater than those of the pure PLA polymer. Likewise, such phenomenon also applied to the sisal fibers-PP biocomposite compared to pure PP polymer. In shaping with the hot-press molding, the microfibrillated sisal fibers-PLA biocomposite and microfibrillated sisal fibers-PP biocomposite that seemed the most prospective for uses and further development were those using the ratio of fibers/polymer at 40/60.

The biocomposite shaped using the injection molding device was conducted only on the one with PLA polymer matrix, using the ratio of fibers/PLA in the range of $10 / 90$ to $30 / 70$. The maximum physical and mechanical properties of microfibrillated sisal fibers-PLA mbiocomposite with such device (i.e. flexural strength/MOR, flexural modulus/MOE, tensile strength, tensile modulus) occurred at the fibers/PLA ratio of $30 / 70$. Such ratio therefore could be regarded as the most prospective in assembling for its uses and further development. MOR and MOE of microfibrillated sisal fibers-PLA biocomposite shaped using the injection molding device at
$30 / 70$ ratio were lower than those of microfibrillated sisal fibers-PLA biocomposite using the hot-press molding also at such ratio. The overall MOR of microfibrillated sisal fibers-PLA biocomposite shaped with such device was lower than that of pure PLA, while its MOE was still mostly higher. The hardness of microfibrillated sisal fibers-PLA biocomposite shaped with the injection molding tended to slightly decrease with increasing portion of fibers in the fibers/PLA ratio. The maximum hardness occurred at 10/90 ratio.

\section{ACKNOWLEDGEMENTS}

The financial support from the Competitive LIPI (Indonesian Institute of Sciences) Program is gratefully acknowledged. The authors would like to appreciate highly the reviewers for suggestion and corections.

\section{REFERENCES}

Arzondo, L. M., Vazquez, A., Carella, J. M., \& Pastor, J. M. (2004). A low-cost, low-fiber breakage, injection molding process for long sisal fiber reinforced polypropylene. Polymer Engineering and Science, 44, 1766-1772.

Berglund, L. M. (2004). Cellulose based nanocomposites. In Mohanty (Ed.), Natural fibers, biopolymers and their biocomposites. CRC Press LCC.

Bledzki, A. K., Faruk, O., \& Sperber, V. E. (2006). Cars from bio-fibres. Macromol. Mater. Eng, 291(5), 449-457.

Bogoeva-Gaceva, G., Avella, M., Malinconico, M., Buzarovska, A., Grozdanov, A., Gentile, G., et al. (2007). Natural fiber ecocomposites. Polymer Composites, 28(1), 98-107. DOI 10.1002/pc.

Iwamoto, S., Nakagaito, N. A., Yano, H., \& Nogi, M. (2005). Optically transparent composites reinforced with plant fiber-based nanofibers. Applied Physics A, 81(6), 1109-1112.

John, M. J., \& Thomas, S. (2005). Biofibres and biocomposites. Carbohydrate Polymer, 71, 343364.

Joseph, M. J., Joseph, K., \& Thomas, S. (1999). Effect of processing variables on the mechanical properties of sisal-fiber reinforced 
polypropylene composites. Composites Science and Technology, 99, 1625-1640.

Li, Y., Mai, Y. W., \& Ye, L. (2000). Sisal fibre and its composites: a review of recent developments. Composite Science and Technology, 60, 2037-2055.

Marsh, G. (2003, April). Next step for automotive materials. Materialstoday, pp. 36-43.

Mathew, A. P., Oksman, K., \& Sain, M. (2005). Mechanical properties of biodegradable composite from poly lactic acid (PLA) and microcrystalline cellulose (MCC). I Applied Polymer Science, 97, 2014-2025.

Mohanty, A. K., Misra, M., \& Drzal, L. T. (2002). Sustainable bio-composites from renewable resources: Opportunities and challenges in the green materials world. J. Polymers and the Environment, 10(1-2), 19-26.

Mohanty, S., Nayak, S. K., Verma, S. K., \& Tripathy, S. S. (2004). Effect of MAPP as coupling agent on the performance of sisal PP composites. Journal of Reinforced Plastics and Composites, 23, 2047 2063.

Mohanty, S., Verma, S. K., Nayak, S. K., \& Tripathy, S. S. (2004). Influence of fiber treatment on the perfor mance of sisal polypropylene composites. Journal of Applied Polymer Science, 94, 1336-1345.

Munawar, S. S. (2008). Properties of non-wood plant fiber bundles and the development of their composites (Disertation). Japan: Department of Forestry and Biomaterials Science, Kyoto University.

Nakagaito, A. N. and H. Yano. (2004). The effect of morphological changes from pulp fiber towards nano-scale fibrillated. Applied Physics A 78: 547-552.

Nakagaito, A., Nakagaito, A. N., \& Yano, H. (2005). The effect of morphological changes from pulp fiber towards nano-scale fibrillated. Applied Physics A, 80, 155-159.

Netravali, A. N., \& Chabba, S. (2003, April). Composites get greener. Materials Today, pp. 2228.

Oksman, K., Skrifvars , M., \& Selin, J. F. (2003). Natural fibers as reinforcement in polylactic cid (PLA) composites. Composites Science and
Technology, 63, 1317-1324.

Suryanegara, L., Nakagaito, A. N., \& Yano, H. (2009). The effect of crystallization of PLA on the thermal and mechanical properties of microfibrillated cellulose reinforced PLA composites. Composites Science and Technology, 69, 1187-1192.

Wambua, P., Ivens, J., \& Verpoest, I. (2003). Natural fibres: can they replace glass in fibre reinforced plastics? Composites Science and Technology, 63, 1259-1264.

Zimmermann, T., Pohler, E., \& Geiger, T. (2004). Cellulose fibrils for polymer reinforcement. Advanced Engineering Materials, 6(9), 754-761. 